\title{
ANÁLISE DA VIABILIDADE DA UTILIZAÇÃO DA CINZA DE BAGAÇO DE CANA- DE-AÇÚCAR COMO SUBSTITUIÇÃO PARCIAL DO CIMENTO PORTLAND
}

\section{Analysis of availability of using sugarcane bagasse ash as partial replacement of Portland Cement}

\section{Bárbara C.S. Lopes ${ }^{1}$, Alexandre Faria Seixo de Britto ${ }^{2}$, Antover Panazzolo Sarmento ${ }^{3}$ Rodrigo Gustavo Delalibera ${ }^{4}$}

Recebido em 24 de setembro de 2014; recebido para revisão em 18 de outubro de 2014; aceito em 10 de novembro de 2014; disponível on-line em 24 de novembro de 2014.

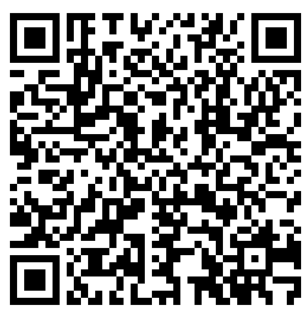

\section{PALAVRAS CHAVE:}

Concreto;

Bagaço de cana-de-açúcar; Resistência à compressão;

\section{KEYWORDS:}

Concrete;

Sugarcane bagasse ash; Compression resistance.

* Contato com os autores:

RESUMO: Utilização de cinza de bagaço de cana-de-açúcar como material suplementar ao cimento Portland, visando minimizar a retirada de calcário, reduzindo a emissão de gás carbônico e preservando jazidas naturais. Foi avaliada a viabilidade do uso parcial de cinza de bagaço de cana-de-açúcar na substituição do cimento Porltand, através da análise de ensaios de resistência à compressão de corpos de prova cilíndricos de concreto aos 7, 14 e 28 dias de idade, apresentando uma substituição em massa de 10, 20 e $30 \%$ do cimento Portland. Considerando-se os resultados de resistência à compressão obtidos verificou-se que é viável a substituição parcial de cinza de bagaço de cana-de-açúcar, desde que a relação água/cimento e a porcentagem em massa a ser substituída sejam adequadas. Deste maneira, espera-se que a utilização da cinza do bagaço de cana-de-açúcar possa interferir economicamente e ecologicamente na produção do concreto.

\begin{abstract}
This paper examines the sugarcane bagasse ash use as supplementary material to Portland cement for the purpose of minimize the limestone removal to reduce emissions carbonic gas preserving natural deposits. The feasibility sugarcane bagasse ash use were analyzed. Through an analysis of results test of cylindrical concrete on 7, 14 and 28 days that showed a 10, 20 and 30 percent weight replacement by Portland cement. The reviewed about this cement and sugarcane bagasse were realized. After that, a experimental study with analysis were realized. Test of granulometry were made in every material that we used for the concrete production. Based on these results, it was concluded that the sugarcane bagasse ash use is feasible, since the water/cement and the mass percentage replaced are appropriate.
\end{abstract}

${ }^{1}$ e-mail : bcs_90@hotmail.com (B. C. S. Lopes )

Engenheira Civil - Profissional autônoma.

2e-mail : alexandre_britto@hotmail.com (A. F. S. Brito)

Graduando do curso de engenharia civil da Universidade Federal do Goiás - Regional Catalão.

${ }^{3}$ e-mail : antoverps@gmail.com (A. P. Sarmento)

Doutor, Professor Adjunto I da Universidade Federal de Goiás - Regional Catalão

${ }^{4}$ e-mail : delalibera@pq.cnpq.br (R.G. Delalibera)

Doutor, Professor Adjunto III da Universidade Federal de Goiás - Regional Catalão. 


\section{INTRODUÇÃO}

Com o crescimento populacional e a elevada demanda de consumo, torna-se importante a necessidade de extração de matérias-primas da natureza. A extração de muitas dessas matérias-primas solicitam grande quantidade de energia e podem emitir gases nocivos ao meio ambiente. Por conseguinte, o aumento na geração de resíduos é inevitável, acarretando a necessidade de áreas cada vez maiores para a disposição final dos mesmos, observando ainda que tal disposição deve atender diversas legislações vigentes.

Observando ainda esse crescimento populacional e o aquecimento econômico nos últimos anos, o qual acarretou um crescimento considerável na construção civil, tem-se gerado um consumo cada vez maior de cimento. O cimento é um dos materiais utilizados em larga escala pela indústria da construção civil; segundo a CETESB (2010) o processo de produção do cimento é resumidamente uma combinação de exploração e beneficiamento de substâncias minerais não metálicas. Durante a fabricação do clínquer, produto intermediário do cimento, é emitido o dióxido de carbono $\left(\mathrm{CO}_{2}\right)$.

A incorporação de resíduos na forma de adição mineral ao cimento promove a redução da quantidade do clínquer por $\mathrm{m}^{3}$ de concreto/argamassa, com isso acarreta a redução dos impactos ambientais e da emissão de $\left(\mathrm{CO}_{2}\right)$, gerados pela indústria do cimento (PAULA, 2006). Com a reciclagem de resíduos, existe a diminuição da necessidade de extração de matérias-primas, do consumo de energia, dos custos de transporte e do volume de aterros; gerando então uma redução do impacto ambiental causado pela construção civil (LIMA, 2007).

A busca de alternativas de materiais para utilização na construção civil não é nova, mas constantemente novas pesquisas e materiais começam a surgir, dentre eles a utilização de cinzas na confecção de concreto e argamassa, à exemplo temos estudos da viabilidade da substituição parcial do cimento por cinza de casca de arroz (NEHDI et al. 2003; DUART, 2008), cinza de casca de castanha de caju (LIMA, 2008), cinza de queima de madeira (JÚLIO et al., 2011) e cinza de bagaço de cana-de-açúcar (VANDERLEl et al., 2014).

Segundo Lima (2008), a indústria da construção civil consome cerca de 20 a $50 \%$ do total de recursos naturais utilizados pelas atividades industriais; por isso o emprego de resíduos na engenharia civil tem se consolidado como uma prática para a sustentabilidade. A utilização de da CBCA implicará na redução da emissão de $\mathrm{CO}_{2}$ quando da produção do cimento Portland, além de dar um destino ao resíduo gerado pela produção de açúcar e álcool.

Dentre as alternativas existentes para atenuar o problema em relação aos resíduos sólidos e a diminuição da retirada de material de jazidas naturais, este trabalho teve por objetivo analisar a utilização da cinza de bagaço de canade-açúcar (CBCA) como substituição parcial do cimento Portland, analisando-se a resistência à compressão de amostras de concreto feitas com teores de substituição de 10, 20 e 30\% e sem substituição do cimento pela CBCA.

\section{MATERIAIS E PROGRAMA EXPERIMENTAL}

\subsection{CINZA DO BAGAÇO DE CANA-DE-AÇÚCAR}

A cinza do bagaço de cana-de-açúcar utilizada no programa experimental é proveniente da usina São Domingos Açúcar e Álcool S.A. com cedo no Município de Catanduva, estado de São Paulo, na qual a caldeira para a queima do bagaço de cana-de-açúcar atinge uma temperatura entre 750 e $800{ }^{\circ} \mathrm{C}$.

Utilizou-se a CBCA in natura, sendo realizado apenas o peneiramento da mesma com uma peneira de malha de $2 \mathrm{~mm}$ para a retirada de material grosseiro, conforme Figura 1. A mesma apresenta granulometria próxima da areia muito fina, e módulo de finura igual a 0,54 de acordo com o ensaio de granulometria, seguindo as recomendações da NBR 7217 (ABNT, 1987). 

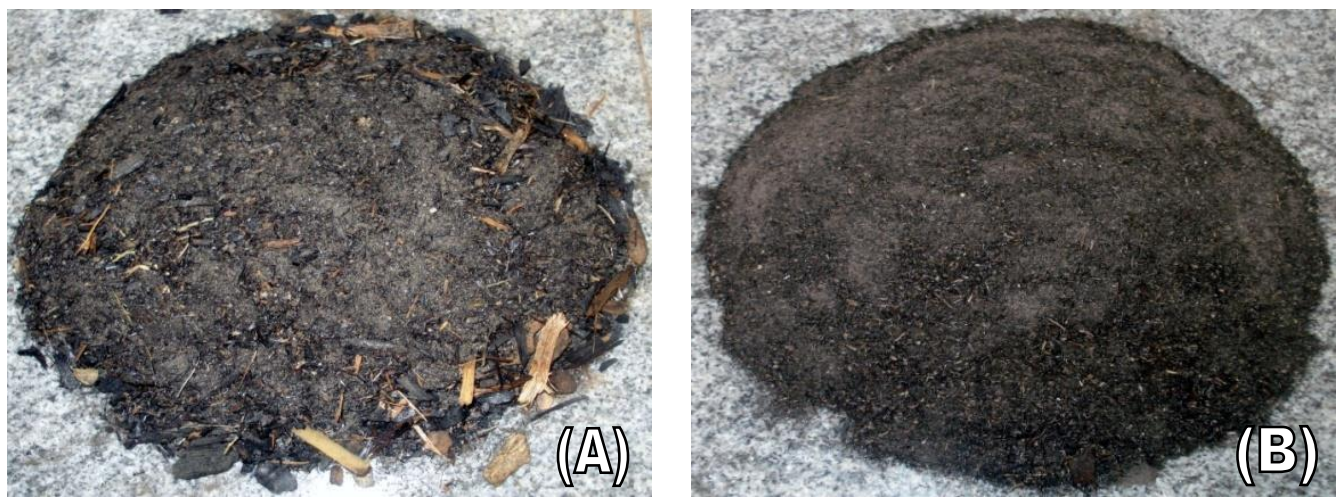

FIGURA 1: (A) CBCA antes do peneiramento e (B) CBCA após o peneiramento.

\subsection{CIMENTO}

O cimento utilizado nos ensaios foi o cimento da marca Ciplan, tipo CP II-Z-32, apresentando granulometria semelhante a da areia muito fina, e módulo de finura igual a 0,70. 0 ensaio de granulometria do cimento seguiu a NBR 7217 (ABNT, 1987).

\subsection{AGREGADO MIÚDO}

O agregado miúdo utilizado foi areia tipo quartzosa, considerada média, pertencendo a zona 3. As características de agregado foram obtidas nos ensaios respeitando-se as indicações NBR 7217 (ABNT, 1987) e NBR 7251 (ABNT, 1982) e, podem ser encontradas na Tabela 1.

TABELA 1: Características do agregado miúdo.

\begin{tabular}{ccc} 
Característica & Norma & Resultado \\
\hline Diâmetro Máx. & $\begin{array}{c}\text { NBR 7217 } \\
\text { (ABNT, 1987) }\end{array}$ & $2,36 \mathrm{~mm}$ \\
Módulo de Finura & $\begin{array}{c}\text { NBR 7217 } \\
\text { (ABNT, 1987) }\end{array}$ & 2,21 \\
Umidade Natural & - & $0,40 \%$ \\
Massa Unitária & $\begin{array}{c}\text { NBR 7251 } \\
\text { (ABNT, 1982) }\end{array}$ & $1,47 \mathrm{~g} / \mathrm{cm}^{3}$ \\
\hline
\end{tabular}

\subsection{AGREGADO GRAÚDO}

O agregado graúdo utilizado foi a pedra britada número 1 cujas características, obtidas nos ensaios respeitando a NBR 7217 (ABNT, 1987) e NBR 7251 (ABNT, 1982) são mostradas na Tabela 2.
TABELA 2: Características do agregado graúdo.

\begin{tabular}{ccc} 
Característica & Norma & Resultado \\
\hline Diâmetro Máx. & $\begin{array}{c}\text { NBR 7217 } \\
\text { (ABNT, 1987) }\end{array}$ & $19 \mathrm{~mm}$ \\
Módulo de Finura & $\begin{array}{c}\text { NBR 7217 } \\
\text { (ABNT, 1987) }\end{array}$ & 1,83 \\
Umidade Natural & - & $0,17 \%$ \\
Massa Unitária & NBR 7251 & $1,64 \mathrm{~g} / \mathrm{cm}^{3}$ \\
& $($ ABNT, 1982) & \\
\hline
\end{tabular}

\subsection{MÉTODO}

Desejando obter uma resistência a compressão aos 28 dias de $25 \mathrm{MPa}$; utilizou-se o método de dosagem de concretos desenvolvido por Helene e Terzian (1995). O método baseia-se em três resultados diferentes, obtidos a partir da produção de três traços com diferentes teores de cimento, definidos como Pobre, Médio e Rico. Fixou-se o teor de argamassa em $\alpha=50 \%$. Por fim obtém-se o resultado final por meio de um diagrama que representa as três leis comportamentais do concreto para uma dada consistência. A partir do estudo do traço anterior e definido o consumo de cimento de cada traço, foi possível determinar a quantidade de $\mathrm{CBCA}$ para cada traço, fixadas as proporções de 10, 20 e 30\% em massa de utilização da CBCA em substituição ao cimento.

Para cada traço (Médio, Rico e Pobre) foram realizados quatro lotes, sendo realizado um lote com a amostra de controle ( $0 \%$ de CBCA) e um para cada porcentagem de substituição de CBCA (10, 20 e 30\% de CBCA em massa). Cada combinação de traço e substituição de CBCA, foi composta por 9 corpos de prova, sendo 
três idades de rompimento (7, 14 e 28 dias) e realizando 3 repetições para cada idade, totalizando 108 corpos de prova.

Os corpos de prova (cilíndricos de $10 \mathrm{~cm}$ de diâmetro por $20 \mathrm{~cm}$ de altura) foram moldados no Laboratório Provisório de Materiais do Departamento de Engenharia Civil da Universidade Federal de Goiás, Regional Catalão.

Inicialmente fez-se a imprimadura da betoneira de eixo inclinado com capacidade para 145 litros utilizando uma mistura contendo areia, cimento e água. Em seguida os materiais foram adicionados à betoneira para a obtenção do concreto fresco a ser utilizado para a moldagem dos corpos de prova. Inicialmente lançou-se a brita e $20 \%$ da água de amassamento, ambas foram misturadas durante um minuto. Em seguida a areia e $50 \%$ do cimento e a CBCA foram adicionadas a mistura, sendo misturados por três minutos, por fim o restante do material foi adicionado e promovida a mistura por quatro minutos. Ao final verificou-se se era possível efetuar o abatimento de tronco de cone, ou seja, se havia coesão e plasticidade adequada.

Os corpos de prova foram retirados do processo de cura e submetidos ao teste de resistência à compressão aos 7, 14 e 28 dias conforme a NBR 5739 (ABNT, 1980). Inicialmente realizou-se a retificação das faces transversais dos corpos de prova e em seguida os mesmos foram submetidos ao ensaio de resistência à compressão.

As médias de resistência à compressão foram analisadas por meio de análise de variância complementando com teste de comparação de médias de Tukey. Os resultados estatísticos foram discutidos a 5\% $(p<0,05)$ de nível de significância. As análises estatísticas foram realizadas utilizandose o software estatístico WinStat 2.11 (MACHADO e CONCEIÇÃO, 2001).

\section{RESULTADOS E DISCUSSÕES}

Para analisar a viabilidade de utilização da CBCA em substituição ao cimento Portland foram ensaiados quatro lotes de concreto, contendo substituições de 0, 10, 20 e 30\% de CBCA "in natura". Optou-se por denominar as amostras conforme a Tabela 3.

Em relação a resistência à compressão analisou-se que a mesma apresentava redução conforme a quantidade de CBCA em substituição ao cimento Portland aumentava, mesmo com o aumento da idade. Aos 28 dias observou-se que redução da resistência ainda foi acentuada onde a substituição de CBCA foi maior que $10 \%$, podendo-se notar que na substituição de $10 \%$, os valores de resistência a compressão foram muito semelhantes ao da amostra controle, principalmente nos traço médio e rico, conforme apresentado nas Figuras 2 (Traço Pobre), 3 (Traço Médio) e 4 (Traço Rico).

TABELA 3: Nomenclatura das amostras.

Traço \% de CBCA Nomenclatura Traço \% de CBCA Nomenclatura Traço \% de CBCA Nomenclatura

\begin{tabular}{|c|c|c|c|c|c|c|c|c|}
\hline Pobre & 0 & PO & Médio & 0 & MO & Rico & 0 & RO \\
\hline Pobre & 10 & P10 & Médio & 10 & M10 & Rico & 10 & R10 \\
\hline Pobre & 20 & P20 & Médio & 20 & M20 & Rico & 20 & R20 \\
\hline Pobre & 30 & P30 & Médio & 30 & M30 & Rico & 30 & R30 \\
\hline
\end{tabular}




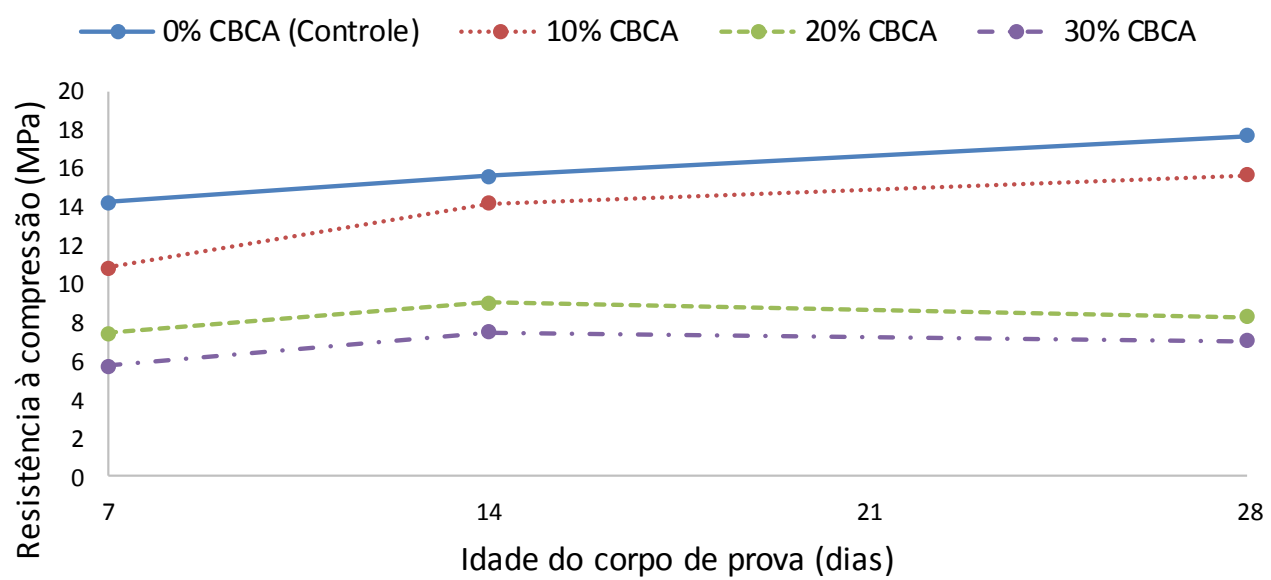

FIGURA 2: Evolução da resistência à compressão das amostras com substituição parcial do cimento Portland por cinza "in natura" - Traço Pobre (Os pontos representam as médias de 3 repetições).

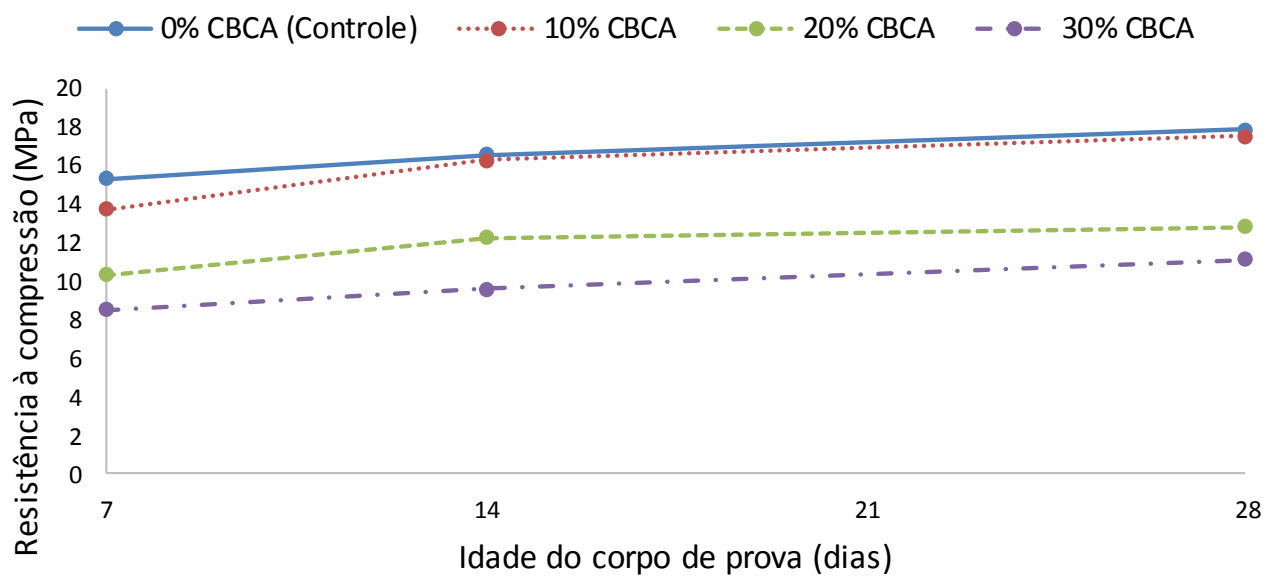

FIGURA 3: Evolução da resistência à compressão das amostras com substituição parcial do cimento Portland por cinza "in natura" - Traço Médio (Os pontos representam as médias de 3 repetições).

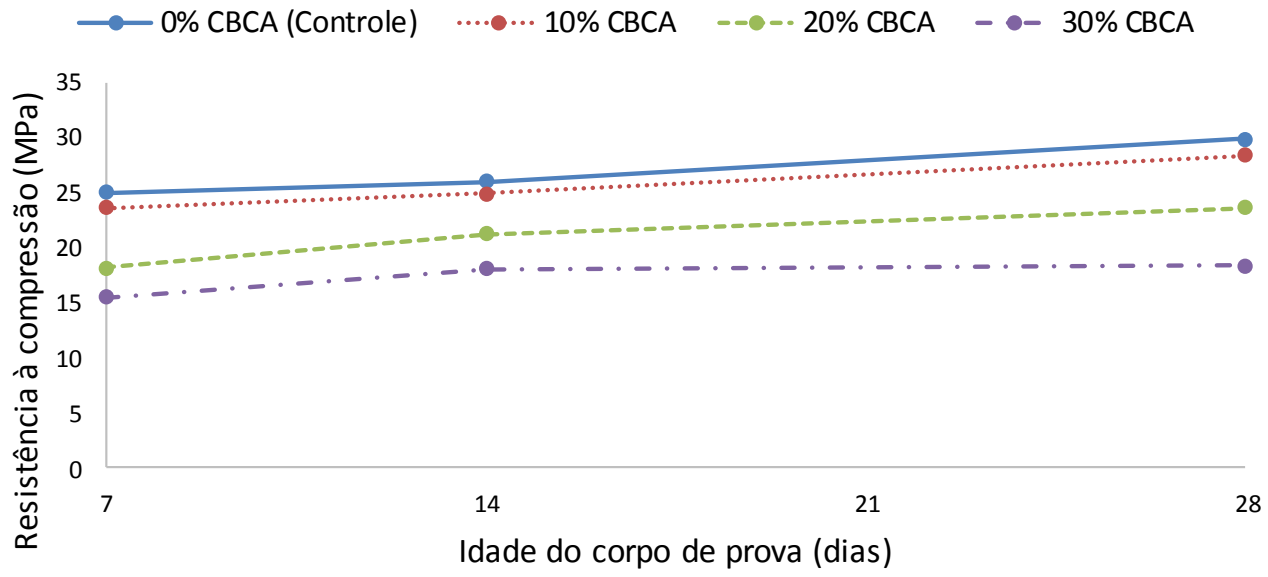

FIGURA 4: Evolução da resistência à compressão das amostras com substituição parcial do cimento Portland por cinza "in natura" - Traço Rico (Os pontos representam as médias de 3 repetições). 
A partir da análise dos dados obtidos neste experimento, constatou-se que a substituição de CBCA em teores de 10\% acarretou uma maior resistência à compressão quando comparada com as outras substituições (20 e $30 \%)$. Contudo, os resultados obtidos no experimento e a análise estatística demonstraram que em qualquer traço, nos 14 e 28 dias de cura, a substituição parcial de cimento Portland por CBCA em teores de $10 \%$ apresentou resistência à compressão estatisticamente semelhante à amostra controle ( $0 \%$ de $C B C A$ ), conforme pode ser observado na Tabela 4.

Observando a Tabela 4, aos 28 dias as amostras P10 apresentaram redução da resistência à compressão em relação a amostra de referência (0\% de CBCA) de 11,47\% (não significativa em nível de $5 \%$ de probabilidade) enquanto as amostras P20 e P30 apresentaram uma redução de 45,37 e 57,89\% respectivamente.

As amostras para 0 traço médio apresentaram menor redução da resistência à compressão quando comparadas as amostras para o traço pobre. Aos 28 dias a redução da resistência à compressão das amostras M10, M20 E M30 em relação a amostra de referência foi de
1,$87 ; 28,32$ e $37,92 \%$ respectivamente.

Já as amostras para o traço rico apresentaram reduções da resistência à compressão aos 28 dias semelhantes as do traço médio. As amostras R10, R20 e R30 em relação a amostra de referência apresentaram redução de 3,10; 19,46 e 37,38\% em sua resistência à compressão aos 28 dias respectivamente.

A partir da análise da redução da resistência à compressão conforme o teor de substituição do cimento Portland pela CBCA constatou-se que o valor da redução da resistência à compressão para o teor de substituição de $30 \%$ apresentou-se muito elevado para os três traços, sendo todos os valores de redução acima de $30 \%$. Em contrapartida para os traços médio e rico, as amostras com teor de substituição de $10 \%$ de CBCA, não houve redução significativa da resistência à compressão.

Verificou-se que as reduções de resistência à compressão mais acentuadas, em qualquer traço, se deram para as substituições de cimento Portland por CBCA nos teores de 20 e $30 \%$, enquanto o teor de $10 \%$ apresentou-se estatisticamente semelhante à referência.

\begin{tabular}{|c|c|c|c|c|c|c|c|c|c|}
\hline Traço & \multicolumn{3}{|c|}{ Pobre $(a / c=0,65)$} & \multicolumn{3}{|c|}{ Médio $(a / c=0,63)$} & \multicolumn{3}{|c|}{$\operatorname{Rico}(a / c=0,45)$} \\
\hline Substituição/Dias & 7 & 14 & 28 & 7 & 14 & 28 & 7 & 14 & 28 \\
\hline $0 \%$ (Controle) & $14,24 \mathrm{a}$ & $15,59 \mathrm{a}$ & $17,70 \mathrm{a}$ & $15,30 \mathrm{a}$ & $16,58 \mathrm{a}$ & $17,87 \mathrm{a}$ & 24,96 a & $26,01 \mathrm{a}$ & $29,83 \mathrm{a}$ \\
\hline $10 \%$ & $10,80 \mathrm{~b}$ & $14,18 \mathrm{a}$ & $15,67 a$ & $13,74 \mathrm{a}$ & $16,29 a$ & $17,54 \mathrm{a}$ & $23,66 \mathrm{ab}$ & $24,91 \mathrm{a}$ & $28,43 a$ \\
\hline $20 \%$ & $7,39 \mathrm{c}$ & $9,01 \mathrm{~b}$ & $8,26 b$ & $10,31 b$ & $12,26 b$ & $12,81 \mathrm{~b}$ & $18,14 a b$ & $21,22 \mathrm{~b}$ & $23,62 b$ \\
\hline $30 \%$ & $5,70 \mathrm{c}$ & $7,45 \mathrm{~b}$ & $7,00 \mathrm{~b}$ & $8,49 \mathrm{~b}$ & $9,58 \mathrm{c}$ & $11,09 \mathrm{~b}$ & $15,44 \mathrm{~b}$ & $18,04 \mathrm{~b}$ & $18,37 \mathrm{c}$ \\
\hline
\end{tabular}


Pode-se observar também que as amostras M10 e R10 demonstraram que a CBCA presente atuou no concreto de modo semelhante a uma adição mineral pozolânica, pois nas primeiras idades, 7 e 14 dias, as resistências à compressão (13,95 e 16,62 MPa) e (24,13 e 25,40 $\mathrm{MPa})$ se mostraram inferiores aos valores encontrados para a amostra M0 (15,61 e 16,91 $\mathrm{MPa})$ e RO (23,75 e 26,53 MPa), entretanto aos 28 dias houve um ganho de resistência à compressão da amostra M10 (17,88 MPa) e da amostra R10 $(28,99 \mathrm{MPa})$, se aproximando da resistência à compressão da amostra MO (18,22 MPa) e da amostra RO (29,91 MPa).

Segundo a NBR 5752 (ABNT, 1992) o índice de atividade pozolânica (IAP) é calculado dividindo a resistência à compressão da amostra contendo o material pozolânico pela resistência a compressão da amostra de controle, ambas aos 28 dias, o resultado final deve ser expresso em porcentagem. De acordo com a NBR 12653 (1992), para uma adição mineral ser caracterizada como pozolânica, a mesma deve apresentar o IAP superior a 75\%. O IAP encontrado para as amostras R10 e M10 foi de $96,92 \%$ e $98,13 \%$ respectivamente, superando os $75 \%$ exigidos pela referida norma, indicando que o ganho de resistência à compressão da amostra R10 e M10 aos 28 dias, aproximando-se da resistência à compressão da amostra de controle RO e MO caracteriza um efeito pozolânico da CBCA utilizada.

De acordo com Mehta e Monteiro (1994) com a idade a resistência da zona de transição do concreto pode tornar-se igual ou maior que a resistência da matriz de argamassa. Esse fato se deve a cristalização de novos produtos nos vazios da zona de transição através de reações químicas lentas, formando silicato de cálcio hidratado. As reações químicas dos óxidos presentes na CBCA com o hidróxido de cálcio formados na hidratação do cimento contribuem para a resistência porque tendem a reduzir a concentração de hidróxido de cálcio na zona de transição.

Como observado por Mehta e Monteiro (1994), a reação pozolânica é lenta, com isso a taxa de liberação de calor e de desenvolvimento da resistência serão consequentemente lentas. A reação também consome óxido, ao invés de produzi-lo, o que representa uma importante contribuição para a durabilidade da pasta endurecida de cimento diante dos meios ácidos.

Analisou-se que apesar do IAP das amostras P10 e R20 apresentarem valor de $88,53 \%$ e $80,54 \%$, superando os $75 \%$ exigidos pela NBR 12653 (1992), a quantidade de silicatos de cálcio hidratado formados pelas reações entre os óxidos presentes na CBCA com o hidróxido de cálcio advindo da hidratação do cimento, não foram suficientes para a resistência à compressão das amostras P10 (15,98 MPa) e R20 (24, $09 \mathrm{MPa})$ superarem ou se aproximarem da resistência à compressão das amostras de referência P0 (18,05 $\mathrm{MPa})$ e RO (29,91 Mpa) aos 28dias.

As amostras P0, P10, P20 e P30 pobres em cimento e também menor quantidade de CBCA, contém traço 1:6,5; portanto sua massa de agregados é maior em relação às demais amostras. A menor quantidade de cimento e CBCA presente nesse traço e maior quantidade de agregados torna o concreto mais poroso e de difícil adensamento. Portanto o concreto se torna menos resistente em virtude dessa maior porosidade em relação às demais amostras. Portanto tais aspectos também são abordados por Mehta e Monteiro (1994), os quais afirmam que além da importância do fator a/aglomerante para a determinação da porosidade tanto da matriz e da zona de transição do concreto, fatores como o adensamento também influenciam no aumento dessa porosidade e consequentemente gera uma redução da resistência à compressão do mesmo.

O ganho de resistência lento, principalmente nas primeiras idades das amostras que apresentaram efeito pozolânico, contendo $\mathrm{CBCA}$, indica um calor de hidratação liberado menor que as amostras sem a presença da cinza. Obras com peças que demandem grande volume de concreto, tendo como consequência elevado calor de hidratação, poderiam lançar mão da utilização da CBCA como adição mineral como alternativa para solucionar o problema com o calor de hidratação gerado nas primeiras idades.

Como citado por Aiticin (2000) a substituição de parte do cimento por um material 
suplementar que possua granulometria bem próxima ou igual a do cimento, torna-se interessante do ponto de vista econômico, pois o preço dos materiais cimentícios suplementares é usualmente menor que o do cimento. No caso deste trabalho a vantagem econômica é ainda mais salientada, pois o material suplementar proposto trata-se de um resíduo, o qual ainda não possui custos, fora o transporte, o que pode resultar na diminuição do custo de produção do concreto, uma vez que teríamos a substituição de $10 \%$ do cimento por um material de menor valor.

A análise da utilização da CBCA como substituição parcial do cimento Portland, tem importância não só sob o aspecto econômico, mas também ambiental, onde os danos acarretados à natureza com a produção de cimento e disposição inadequada dessas cinzas são minimizados pela substituição parcial do cimento pela CBCA. Entretanto a utilização da CBCA em concretos para ser considerada viável deve levar em consideração além de aspectos ambientais e econômicos, os aspectos técnicos como a resistência à compressão do concreto, durabilidade, fluência, retração, reação álcali-agregado etc. Portando, novas alternativas de materiais devem ser pesquisadas. Visando solucionar tais problemas a utilização de resíduos de processos industriais como matérias-primas, torna-se uma prática de sustentabilidade.

\section{CONCLUSÕES}

Este trabalho conteve-se em analisar a resistência à compressão de concretos utilizando CBCA com substituição parcial de 10, 20 e $30 \%$ em massa do cimento Portland II-Z-32. Analisou-se que tal substituição é viável, entretanto a substituição de cimento Portland por CBCA deve estar situada na faixa de 0 a $10 \%$ para que a resistência à compressão não seja afetada.

É preciso esclarecer que este trabalho foi uma investigação preliminar sobre a utilização da CBCA, e que ainda deverão ser realizados estudos mais aprofundados para verificação da viabilização técnica e econômica deste resíduo para seu confiável emprego como material de construção, visando atingir fatores econômicos e ambientais com relação ao uso do CBCA na produção do concreto.

\section{AGRADECIMENTOS}

Os autores gostariam de agradecer ao Sr. Sérgio Vaqueiro da Usina São Domingos de - Açúcar e Álcool S.A pelo fornecimento das cinzas de bagaço de cana-de-açúcar.

\section{REFERÊNCIAS BIBLIOGRÁFICAS}

AÏTICIN, P. C. Concreto de Alto Desempenho. São Paulo: PINI. 2000. 667p.

ASSOCIAÇÃO BRASILEIRA DE NORMAS TÉCNICAS (1987). NBR 7217:1987 - Agregados - determinação da composição granulométrica. Rio de Janeiro;

ASSOCIAÇÃO BRASILEIRA DE NORMAS TÉCNICAS (1982). NBR 7251:1982 Agregados em estado solto determinação da massa unitária. Rio de Janeiro;

ASSOCIAÇÃO BRASILEIRA DE NORMAS TÉCNICAS (1980). NBR 5739:1980 - Ensaio de compressão de corpos-deprova cilíndricos de concreto. Rio de Janeiro.

ASSOCIAÇÃO BRASILEIRA DE NORMAS TÉCNICAS (1992). NBR 5752:1992 Materiais pozolânicos - Determinação de atividade pozolânica com cimento Portland - Índice de atividade pozolânica com o cimento- Método de ensaio. Rio de Janeiro.

ASSOCIAÇÃO BRASILEIRA DE NORMAS TÉCNICAS (1992). NBR 12653:1992 Materiais pozolânicos. Rio de Janeiro.

CETESB - Companhia Estadual de Tecnologia de Saneamento Ambiental. Inventário de emissões de gases de efeito estufa associados aos processos industriais: produtos minerais, produção de cimento do estado de São Paulo, 1990 a 2008. Relatório técnico. São Paulo: CETESB, 2010. 27 p.

DUART, M. A. Estudo da microestrutura do concreto com adição de cinza de casca de arroz residual sem beneficiamento. 2008. 134 f. Dissertação (Mestrado em Engenharia Civil). Universidade Federal de Santa Maria, Santa Maria, 2008.

HELENE, P.; TERZIAN, P. R. Manual de dosagem e controle do concreto. São Paulo, PINI. 1995. 
JULIO, A. L., GRUB, J., BAGNARA, D., DIPP, G.H., ABREU, A. G. Utilização de cinzas provenientes da queima de madeira em caldeira de agroindústria .In: VI SIMPÓSIO DE ENGENHARIA AMBIENTAL DO ESPIRITO SANTO, 9., 2011, Vitória. Anais... FAESA, 2011.

LIMA, R. G. C. Resíduos industriais- Método de tratamento e análise de custos. 2007. $18 \mathrm{f}$. Trabalho de Conclusão de Curso (Graduação em Engenharia Ambiental). Curso de Graduação em Engenharia Ambiental, Universidade Católica de Goiás, Goiânia, 2007.

LIMA, S. A. Análise da viabilidade do uso de cinzas agroindustriais em matrizes cimentícias: Estudo de caso da cinza da casca da castanha de caju. 2008. $160 \mathrm{f}$. Dissertação (Mestrado em Arquitetura e Urbanismo) Escola de Engenharia de São Carlos, Universidade de São Paulo, São Carlos, 2008.

MACHADO, A. A.; CONCENIÇÃO, A. R. WinStat Sistemas de análises estatísticas para Windows. Versão 2.11. UFPel - Universidade Federal de Pelotas, NIA - Núcleo de Informática Aplicada, 2011.

MEHTA, P. K., MONTEIRO, P. J. M. Concreto: Estrutura, Propriedades e Materiais. São Paulo: PINI. 1994. 573p.

NEHDI, M., DUQUETE, J., DAMATTY, E. L., Performance of Rice husk ash produced using a new tecnology as a mineral admixture in concrete. Cemente and Concrete Research, v.33, n.8, p. 1203-1210. 2003.

PAULA, M. O. Potencial da cinza do bagaço da cana-deaçúcar como material de substituição parcial de cimento Portland. 2006. 77 f. Dissertação (Mestrado em Engenharia Agrícola), Universidade Federal de Viçosa, Viçosa, 2006.

VANDERLEI, R. D. PEINADO, H.S.; NAGANO, M.F.; DAL MOLIN FILHO, R.G. Cinza do bagaço de cana-de-açúcar como agregado em concretos e argamassas. REEC Revista Eletrônica de Engenharia Civil, v. 8, n. 1, mar. 2014. 